\title{
Performance analysis of quadratic congruence codes using superstructured fiber Bragg gratings for a flexible data rate coherent DS-OCDMA system
}

\author{
Ihsan Fsaifes,,${ }^{1,2, *}$ Catherine Lepers, ${ }^{2}$ Renaud Gabet, ${ }^{1}$ Marc Douay, ${ }^{2}$ and \\ Philippe Gallion ${ }^{1}$ \\ ${ }^{1}$ Institut TELECOM, TELECOM ParisTech, Laboratoire Traitement et Communication \\ de l'Information, CNRS, UMR 5141, Ecole Nationale Supérieure des \\ Télécommunications, 46 Rue Barrault, 75013, France \\ ${ }^{2}$ Laboratoire de Physique, des Lasers, Atomes et Molécules, CNRS, UMR 8523, \\ Université de Lille, 59655 Villeneuve d'Ascq, France \\ *Corresponding author: Ihsan.Fsaifes@phlam.univ-lillel.fr
}

\begin{abstract}
Received January 28, 2008; revised May 28, 2008; accepted May 28, 2008; published June 27, 2008 (Doc. ID 92203)

The performance of a coherent direct sequence optical code division multiple access (DS-OCDMA) system using quadratic congruence codes implemented with superstructured fiber Bragg gratings is experimentally analyzed. We point out that nonperiodic and sparse quadratic congruence codes reduce multipath beat noise and intersymbol interference, respectively. These two properties of the structure of the quadratic congruence codes are shown to improve the bit-error-rate (BER) performance of the coherent system. As a result, we demonstrate that the performance enhancement of the coherent DS-OCDMA system allows the data bit rate of the system to be varied from $1 \mathrm{Gbit} / \mathrm{s}$ to $2.5 \mathrm{Gbits} / \mathrm{s}$ and the receiver bandwidth to be decreased from $15 \mathrm{GHz}$ to $5 \mathrm{GHz}$ respecting the low-cost requirement of the optical access network. These data bit rate and receiver bandwidth performances are achieved with moderate BER penalty. (C) 2008 Optical Society of America

OCIS codes: $060.2330,060.2340,060.4230$.
\end{abstract}

\section{Introduction}

Renewed interest has been paid to optical code division multiple access (OCDMA) for metropolitan and local area network applications [1]. Today, the traffic is highly granular with hundreds of users pushing data and services leading to higher data bit rate needs. A variety of OCDMA architectures are currently under investigation targeting these applications [2-5].

OCDMA system performance is strongly related to the nature of the optical source type involved in the design of the system. Depending on the source coherence time, the OCDMA system can be coherent or incoherent. Coherent OCDMA systems involve using optical laser sources for which the coherence time $\tau_{c}$ is very large compared with the integration time duration $T_{D}$ of the photodetector. Using broadband sources leads to incoherent OCDMA systems because the ratio $\tau_{c} / T_{D}$ is small compared with 1 .

The choice of an incoherent or direct detection scheme is commonly used in OCDMA systems. Due to the square-law detection process, intensity addition of optical signals and the beating signal between different optical signals can be observed at the photodetector. The beating signal is considered as spurious in the network and is usually called beat noise. Optical source noise, beat noise, and multiple access interference strongly influence the transmission quality of OCDMA systems [6-8].

In previous work, we showed that superstructured fiber Bragg grating (S-FBG) encoders and decoders for a direct sequence OCDMA (DS-OCDMA) system act as multipath interferometers. This behavior together with the coherence of the DS-OCDMA system let the encoded and decoded pulses remain phase correlated and leads to an additional source of noise called multipath beat noise as the pulses superimpose within one pulse chip time duration $T_{c}$ on the quadratic detector. Furthermore, we have demonstrated that coherence time impairments are enhanced when using codes 
with a periodic structure. A periodic code sequence has the same 0 chip number between two successive 1 chips. To overcome these impairments, we suggested the use of sparse and nonperiodic codes, such as quadratic congruence codes (QCs) and extended quadratic congruence codes (EQCs) [9]. Using these codes, pulses from multiple reflections in the encoders and decoders do not combine systematically with pulses from primary reflections within one pulse chip time duration and consequently do not interfere. This leads to a DS-OCDMA system less sensitive to coherence time impairments [10].

In this work, we investigate experimentally the performance of encoders and decoders using sparse and nonperiodic quadratic congruence codes in the presence of multipath beat noise (MBN), intersymbol interference (ISI), and multiple access interference (MAI). The paper is organized as follows: in Section 2, we describe the architecture of an OCDMA system and its associated noises. In Section 3, we present the realization of the encoders and decoders using the Lloyd mirror interferometer method, and we explain how mismatches between encoders and decoders can be overcome. We then analyze the measured encoder and decoder temporal responses. In Section 4 , we investigate experimentally the effect of multipath beat noise, intersymbol interference on the bit-error-rate (BER) performance of the flexible data bit rate coherent DS-OCDMA system using sparse and nonperiodic extended quadratic congruence codes. We also analyze the effect of the performance enhancement on data bit rate and receiver bandwidth. Finally, we provide some useful discussions and conclusions in Section 5.

\section{OCDMA System Architecture with Associated Noise}

The performance analysis of an OCDMA system requires a complete investigation of all relevant noise sources. Two proper noise sources are related to the OCDMA technique and are identified as beat noise due to quadratic direct detection and MAI. MAI result from the presence of multiple users in the network that are unable to decode correctly the transmitted encoded data if they do not possess the exact code sequence; they are so called interferers. Other noises, such as shot noise and receiver thermal noise, also have to be considered (Fig. 1).

In [8], the authors analyzed the effect of beat noise and other additive noises in OCDMA systems by defining the coherent ratio between the chip time duration $T_{c}$ to the source coherence time $\tau_{c}$. Using direct photodetection with integration time duration $T_{D}$ limited to the chip time duration $T_{c}$, the output signal $Z$ after integration is given by

$$
\begin{aligned}
& Z=\underbrace{T_{c} \Re P_{d}}_{\begin{array}{c}
\text { Desired } \\
\text { signal }
\end{array}}+\underbrace{T \Re \sum_{i=1}^{m} P_{i}}_{\text {MAI }} \\
& +\underbrace{2 \Re \sum_{i=1}^{m} \sqrt{P_{d} P_{i}} \int_{0}^{T_{c}} \cos \left[\left(\omega_{i}-\omega_{d}\right) t-\omega_{i} \tau_{i}+\phi_{i}\left(t-\tau_{i}\right)-\phi_{d}(t)\right] \mathrm{d} t} \\
& \text { User-interferer beatings } \\
& +\underbrace{2 \Re \sum_{j=i+1}^{m} \sum_{i=1}^{m-1} \sqrt{P_{i} P_{j}} \int_{0}^{T_{c}} \cos \left[\left(\omega_{i}-\omega_{j}\right) t-\omega_{i} \tau_{i}+\omega_{j} \tau_{j}+\phi_{i}\left(t-\tau_{i}\right)-\phi_{j}\left(t-\tau_{j}\right)\right] \mathrm{d} t} \\
& \text { Interferer-interferer beatings } \\
& +\underbrace{\int_{0}^{T_{c}} n_{0}(t) \mathrm{d} t}_{\text {Receiver noise }}
\end{aligned}
$$

where $P_{d}$ and $P_{i}$ are, respectively, the optical intensity of the correctly decoded signal from the desired user and the incorrectly decoded signals from the undesired users (interferers), $\omega_{d}$ and $\omega_{i}$ are optical frequencies, and $\phi_{d}$ and $\phi_{i}$ are the phase noises of these signals. $\tau_{i}$ is the delay time between the desired signal and the interferers, and $\mathfrak{R}$ is the responsitivity of the photodetector. 


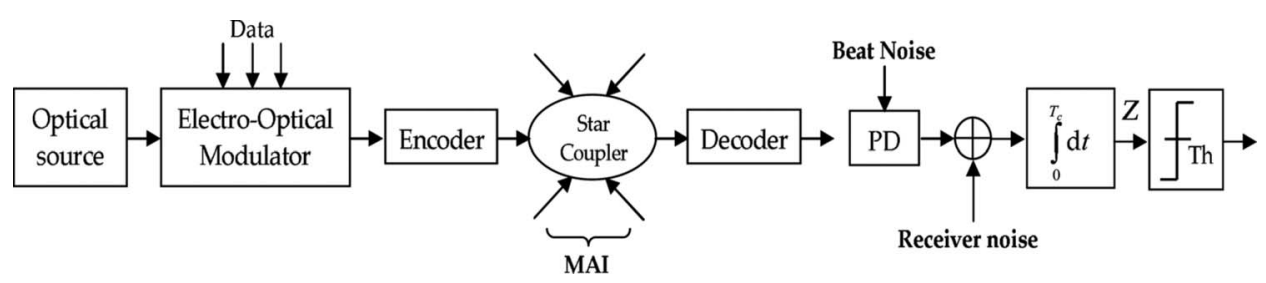

Fig. 1. OCDMA system architecture with different noise sources.

The first term in Eq. (1) represents the correctly decoded signal (desired signal). The second term represents the MAI. The third and fourth terms represent, respectively, the $m$ user-interferer beating signals between the desired signal and the interferer signals and the $m(m-1) / 2$ beating signals between interferers in the network. The last term corresponds to the receiver noise.

Assuming that the desired signal and the interferers are transmitted from the same optical source, in this case $\omega_{d}-\omega_{i}=0$. When the number of users sharing the network is low, frequency beating between interferers can be neglected. Equation (1) becomes

$$
\begin{aligned}
Z= & \underbrace{T_{c} \Re P_{d}}_{\begin{array}{c}
\text { Desired } \\
\text { signal }
\end{array}}+\underbrace{T_{c} \Re \sum_{i=1}^{m} P_{i}}_{\text {MAI }} \\
& +\underbrace{2 \Re \sum_{i=1}^{m} \sqrt{P_{d} P_{i}} \int_{0}^{T_{c}} \cos \left[-\omega_{i} \tau_{i}+\phi_{i}\left(t-\tau_{i}\right)-\phi_{d}(t)\right] \mathrm{d} t}_{\text {User-interferer beatings }} \\
& +\underbrace{\int_{0}^{T_{c}} n_{0}(t) \mathrm{d} t .}_{\text {Receiver noise }}
\end{aligned}
$$

As has been noted in [8], the term $\delta \phi_{i, d}(t)=\phi_{i}\left(t-\tau_{i}\right)-\phi_{d}(t)$ strongly depends on the source coherence time $\tau_{c}$. We discuss hereafter the effect of this term in the case of incoherent and coherent OCDMA systems, and we introduce the new source of noise multipath beat noise observed in the system considered in this work.

Incoherent system when $\tau_{C} \ll T_{D}$. For incoherent OCDMA systems, broadband sources are commonly used. The source coherence time is very small compared with the integration time duration of the photodetector $T_{D}$. In this case, the term $\delta \phi_{i, d}(t)$ is a random process uniformly distributed over $[-\pi, \pi]$. The beating signal between the desired user and the interferers is neglected. Equation (2) becomes

$$
Z=T_{c} \Re P_{d}+T_{c} \Re \sum_{i=1}^{m} P_{i}+\int_{0}^{T_{c}} n_{0}(t) \mathrm{d} t .
$$

The main noise source is the MAI. The autocorrelation function may be considered the sum of the different optical powers of all pulses to be combined within one chip time duration.

In the particular case, when the broadband source is a coherent supercontinuum, the beating signal between the desired user and the interferers cannot be neglected. Equation (2) is then still obvious for this case [11].

Coherent system when $\tau_{C} \geqslant T_{D}$. In this case, coherent laser sources are used. The source coherence time is larger than the integration time duration of the photodetector $T_{D}$. Equation (2) becomes

$$
Z=T_{c} \Re P_{d}+T_{c} \Re \sum_{i=1}^{m} P_{i}+2 \Re \sum_{i=1}^{m} \sqrt{P_{d} P_{i}} \int_{0}^{T_{c}} \cos \left[-\omega_{i} \tau_{i}+\delta \phi_{i, d}(t)\right] \mathrm{d} t+\int_{0}^{T_{c}} n_{0}(t) \mathrm{d} t .
$$

The term $\delta \phi_{i, d}(t)=\phi_{i}\left(t-\tau_{i}\right)-\phi_{d}(t)$ is constant over $[-\pi, \pi]$ during the integration time duration of the photodetector $T_{D}$ and gives rise to an incoherent signal or a coherent beating signal between the desired user and interferers: 
- incoherent signal if the delay $\tau_{i}$ is larger than the coherence time of the laser, $\tau_{i}$ $>\tau_{c}$.

- coherent beating signal if the delay $\tau_{i}$ is lower than the coherence time of the laser, $\tau_{i}<\tau_{c}$.

In the case of a DS-OCDMA system, the code is composed of temporal chips. If the OCDMA system is incoherent, Eq. (4) can be expressed as

$$
Z=\underbrace{T_{c} \Re \omega P}_{\substack{\text { Desired } \\
\text { signal }}}+\underbrace{T_{c} \Re \lambda_{c} m P}_{\text {MAI }}+\underbrace{\int_{0}^{T_{c}} n_{0}(t) \mathrm{d} t}_{\begin{array}{c}
\text { Receiver } \\
\text { noise }
\end{array}},
$$

where the weight of the code $\omega$ presents the number of chips to 1 to be combined within a chip time duration to construct the autocorrelation function. $\lambda_{c}$ is the maximum level of the cross-correlation function. In this case, the autocorrelation function is considered the sum of the $\omega$ power pulses to be combined within $T_{c}$.

When the DS-OCDMA system is coherent, Eq. (4) can be expressed as

$$
\begin{aligned}
Z= & \underbrace{T_{c} \Re\left|\sum_{n=1}^{\omega} e^{j \phi_{n}}\right|^{2}}_{\begin{array}{c}
\text { Multipath } \\
\text { beat noise }
\end{array}}+\underbrace{T_{c} \Re m \lambda_{c} P}_{\text {MAI }} \\
& +\underbrace{2 \Re \sum_{i=1}^{m} \sqrt{P_{d} P_{i}} \int_{0}^{T_{c}} \cos \left[-\omega_{i} \tau_{i}+\delta \phi_{i, d}(t)\right] \mathrm{d} t}_{\text {User-interferer beatings }} \\
& +\underbrace{\int_{\text {noise }}^{\int_{c} n_{0}(t) \mathrm{d} t} .}_{\text {Receiver }}
\end{aligned}
$$

We note that the phase term $\phi_{n}$ is constant during the integration time duration of the photodetector $T_{D}$, which leads to a beating signal between multiple pulses that superimpose within one chip time duration to construct the autocorrelation function. This beating signal concerns one user and will be called hereafter multipath beat noise $(\mathrm{MBN})$ or user-to-user beating signal and is strongly related to the periodicity of the considered codes.

\section{Encoder and Decoder Analysis}

The key components of OCDMA systems are the encoder and decoder that perform code generation and data recognition, respectively. The performance of OCDMA systems is strongly related to the correlation properties of the code family used. A given code family must provide good correlation properties, i.e., high autocorrelation main peak with low out-of-phase sidelobes and low cross-correlation function lobes to reduce MAI between interferers and the desired user. In [9], we demonstrated that $\mathrm{QC}$ and EQC codes have better correlation properties than prime sequences (PSs). These properties are partly due to the sparser of the codes. It is noticeable that the out-of-phase sidelobes of a PS autocorrelation function can be as high as $p-1$, whereas $\mathrm{QC}$ and $\mathrm{EQC}$ codes have been designed to avoid these high autocorrelation sidelobes. They take a maximum value of $\lambda_{a}=2$ for $\mathrm{QC}$ and $\lambda_{a}=1$ for EQC. The crosscorrelation peaks take a value of $\lambda_{c}=4$ for QC in the worst case (when $p>7$ ). They are limited to a maximum value of $\lambda_{c}=2$ for EQC as for PS codes.

In the following subsections, we describe the realization of the encoder and decoder implementing $\mathrm{QC}$ and EQC codes, and we analyze their temporal responses.

\section{A. Encoder and Decoder Realization}

Encoder and decoder realization is achieved by UV photoinscription of superstructured fiber Bragg gratings with the Lloyd mirror interferometer technique. The UV 
source is a frequency-doubled cw argon-ion laser working at $\lambda_{\mathrm{uv}}=244 \mathrm{~nm}$. The Lloyd interferometer consists of a dielectric mirror that reflects part of the incident UV beam to a fiber perpendicular to the mirror. The fringe patterns are formed by the interference between the reflected and the unreflected parts of the incident UV beam. The period of the grating is given by $\Lambda=\lambda_{\text {uv }} / 2 \sin \varphi$, where $\varphi$ is the half-angle between the intersection of UV beams. The width of the beam is adjusted such that the grating length is $L_{\mathrm{FBG}}=830 \mu \mathrm{m}$, corresponding to a FWHM bandwidth of $\Delta \lambda_{\mathrm{FBG}}=1 \mathrm{~nm}$.

The encoder and decoder are written at an initial Bragg wavelength of $\lambda_{\mathrm{Bi}}$ $=1551.7 \mathrm{~nm}$. Optical fiber has been hydrogen loaded to improve the photoinscription process. Under those conditions, the Bragg wavelength undergoes a shift toward a lower wavelength, which has been measured to be around $1 \mathrm{~nm}$ when all the residual molecular hydrogen in the encoder and decoder is outdiffused. This means that the final Bragg wavelength will be $\lambda_{\mathrm{Bf}} \approx 1550.7 \mathrm{~nm}$. In the BER experimental setup, the distributed-feedback (DFB) laser source will be tuned to this wavelength $\lambda_{\mathrm{Bf}}$ to get a maximum mean reflectivity of $16 \%$ for PS and EQC codes $(p=3)$ and $10 \%$ for QC codes $(p=5)$.

To overcome Bragg wavelength mismatch between fiber Bragg gratings inside the encoder and the decoder, index modulations have been chosen to be as close as possible by increasing the length of fiber Bragg gratings to $L_{\mathrm{FBG}}=830 \mu \mathrm{m}$ instead of $L_{\mathrm{FBG}}=455 \mu \mathrm{m}$ used in previous work. The reflectivity of each fiber Bragg grating has been achieved thanks to the kinetics curve. Matching Bragg wavelengths and achieving nominal reflectivities of fiber Bragg gratings permit one to obtain pulses presenting the same reflected mean optical power in the encoder and decoder temporal responses in order to optimize the signal-to-noise ratio at the photodetector $[12,13]$.

\section{B. Experimental Encoder and Decoder Temporal Responses}

Simulated and experimental temporal responses of encoder, decoder, and autocorrelation function for nonperiodic prime sequence and extended quadratic congruence codes with $p=3$ are given in Fig. 2. A good agreement can be observed between simulations and experiments. First, we observe that, in the encoder and matched decoder temporal responses, the optical pulses present the same instantaneous maximum optical power, and multiple reflections do not affect the amplitude level of primary pulses anymore. In [10], using PS periodic code structure (100010001), a beating signal was observed between the third primary pulse signal and the secondary reflected pulse signal, which are superimposed in the same chip time duration. In this case, the third pulse of the code exhibited different optical power levels depending on the polarization state of the system.

Second, the main peak of the autocorrelation function is surrounded with weak sidelobes. Sidelobes on the left side of the autocorrelation peak do not interfere with other pulses. Lower powerful sidelobes can be observed on the right side. This feature is due to beat noise involved by the multipath interferometer behavior of the encoder and the decoder $(\mathrm{MBN})$. Therefore, these interactions due to source coherence time are less critical when using nonperiodic code structure.

We have designed and realized an encoder and a decoder for QC codes with $p=5$. In this case, the number of Fabry-Perot cavities limited by the different fiber Bragg gratings inside the encoder and decoder increases and leads to further multipath reflection pulses. Achieving such an encoder and a decoder with the same mean reflected optical power for each pulse is more difficult because it needs a very close linear kinetics curve to achieve the nominal fiber Bragg grating reflectivities (Fig. 3).

The nonperiodicity of $\mathrm{QC}$ and $\mathrm{EQC}$ codes leads to an all-optical CDMA system that is less sensitive to source coherence time impairments. The multiple reflection signals from the different Fabry-Perot coupled cavities do not sum systematically with the main pulses in one chip time duration as is observed for periodic codes. However, the reconstructed autocorrelation function after decoding has still to be considered in a complex amplitude basis, even if it is more robust against MBN.

In Section 4, we study the performance of a coherent DS-OCDMA system using nonperiodic codes implemented with superstructured fiber Bragg gratings. We demonstrate experimentally the effect of multipath beat noise and intersymbol interference impairments on bit-error-rate performance by sweeping the data rate of the system from $1 \mathrm{Gbit} / \mathrm{s}$ to $2.5 \mathrm{Gbits} / \mathrm{s}$. By comparing the results with those of periodic codes, we 

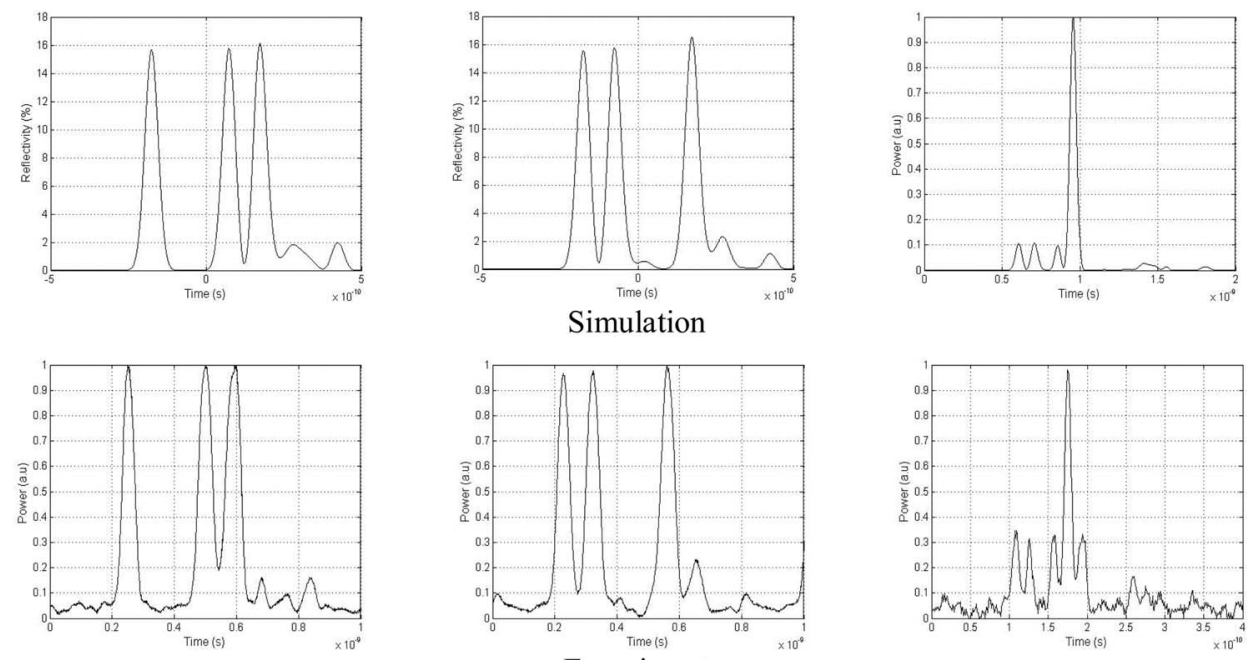

Experiment

(a)
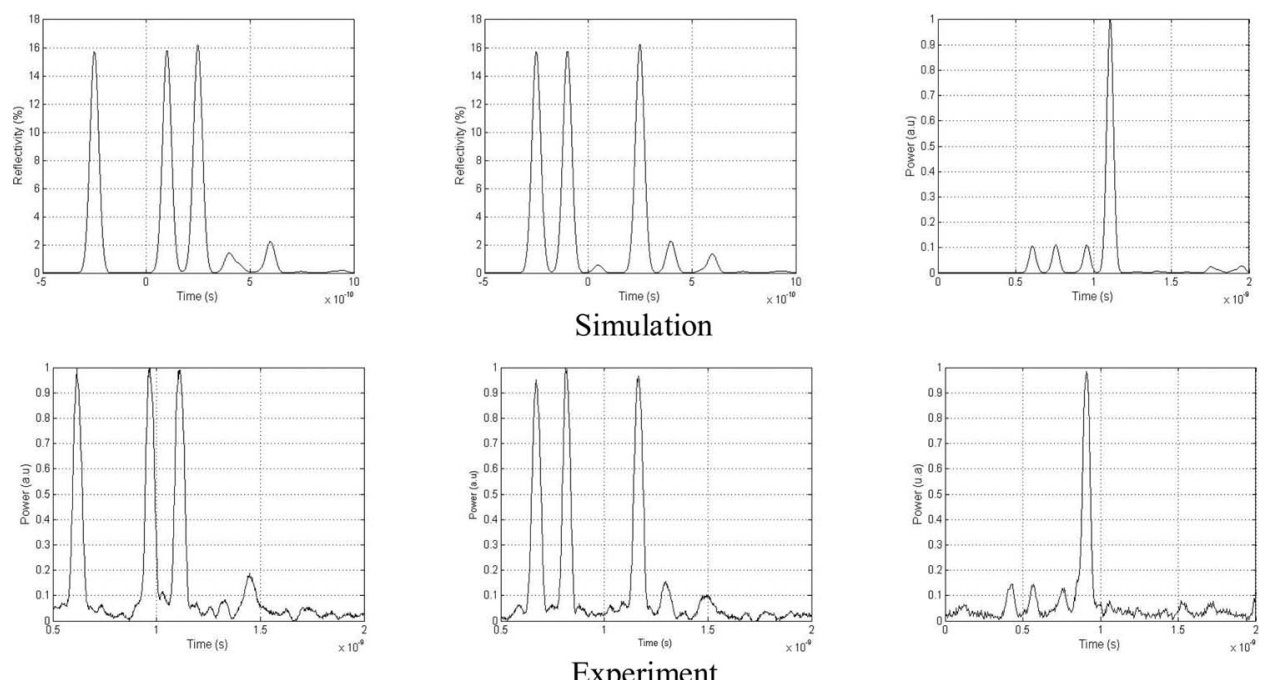

Experiment

(b)

Fig. 2. Simulated and experimental temporal responses of encoder, decoder, and autocorrelation function for nonperiodic codes: (a) PS ( $p=3)$, (b) EQC $(p=3)$.

demonstrate that sparse and nonperiodic codes improve the system performance against beat noise and ISI.

\section{Bit-Error-Rate Experimental Setup and System Performance}

The BER experimental setup consists of a $1550.7 \mathrm{~nm}$ DFB laser with an integrated electro-absorption modulator (ILM) driven by a $10 \mathrm{GHz}$ clock signal allowing the laser to operate in a pulse regime. The resulting $50 \mathrm{ps}$ pulse train is return-to-zero (RZ) modulated by an electro-optic modulator (EOM 1) at a $1 \mathrm{Gbit} / \mathrm{s}$ data rate per user (with a pulse ratio of $1: 10$ ). The $1 \mathrm{Gbit} / \mathrm{s}$ signal is then modulated with a pseudorandom binary sequence (PRBS) signal by a second electro-optic modulator (EOM 2). The resulting pulse train is then amplified and separately encoded by encoder 1 and encoder 2 . The data streams are decorrelated using a delay line avoiding multipleuser beat noise at the photodetector. The resulting signals are then transmitted to the matched decoder (decoder 1). At the detection, a variable attenuator is used to control the received optical power during the BER measurements (Fig. 4).

4.A. Intersymbol Interference and Multipath Beat Noise

MAI is usually considered the main degradation in OCDMA systems. In this subsection, we show that $\mathrm{MBN}$ related to the design of the encoder and decoder, together 

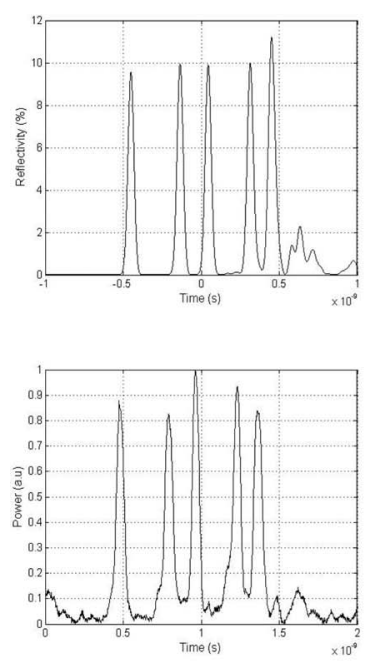

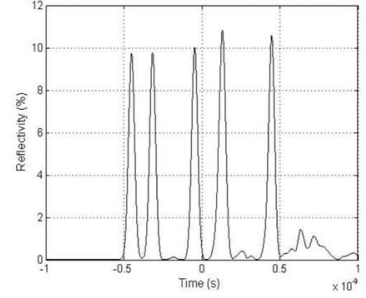

Simulation

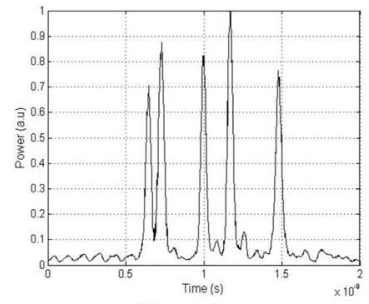

Experiment
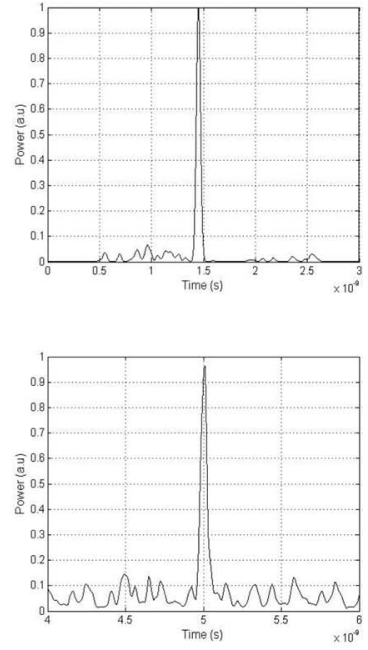

Fig. 3. Simulated and experimental temporal responses of encoder, decoder, and autocorrelation function for QC $(p=5)$ nonperiodic code.

with the coherence properties of the laser source, can also limit the performance of the system. However, we have seen before that these effects depend on the choice of the codes. We also study the effect of ISI by sweeping the data rate from $1 \mathrm{Gbit} / \mathrm{s}$ to $2.5 \mathrm{Gbits} / \mathrm{s}$.

In a DS-OCDMA system, the encoded pulse duration is $T_{\text {code }}\left(T_{\text {code }}=L T_{c}\right.$, where $L$ is the code length), and the matched and unmatched decoded pulse time duration (corresponding to the autocorrelation and cross-correlation functions, respectively) is $2 T_{\text {code }}$. To avoid ISI, the bit time duration $T_{\text {bit }}$ must be larger than $2 T_{\text {code. When }} T_{\text {bit }}<2 T_{\text {code }}$, ISI occurs in the decoded data streams. Decoded signals for different data bit rates ( $1 \mathrm{Gbit} / \mathrm{s}, 1.25 \mathrm{Gbits} / \mathrm{s}$, and $2.5 \mathrm{Gbits} / \mathrm{s}$ ) are presented in Fig. 5: the OCDMA system uses a nonperiodic PS code in one user configuration with a code length equal to $L$ $=9$. The code time duration is $T_{\text {code }}=450 \mathrm{ps}$. MBN is observed when primary and multipath pulses superimpose within a chip time duration. MAI are not considered here as no interferers are considered. For a $1 \mathrm{Gbit} / \mathrm{s}$ data bit rate, the decoded pulses are largely separated and the ISI does not affect the system. When the data bit rate varies from $1.25 \mathrm{Gbit} / \mathrm{s}$ to $2.5 \mathrm{Gbit} / \mathrm{s}$, ISI increases and different pulses are able to beat together giving rise to beat noise (BN) [Fig. 5(a)]. A BER measurement of $10^{-9}$ is obtained for $1 \mathrm{Gbit} / \mathrm{s}$ and $1.25 \mathrm{Gbits} / \mathrm{s}$ data bit rates. For a $2.5 \mathrm{Gbits} / \mathrm{s}$ data bit rate, a BER of $10^{-8}$ is obtained with a $6 \mathrm{~dB}$ power penalty due to additional ISI [Fig. 5(b)]. For higher data bit rates, ISI would become more critical and would result in worse BER penalties.

Using sparse and nonperiodic EQC codes with a $L=15$ code length, MBN and ISI noise effects are observed to be much less important than with nonperiodic PS code

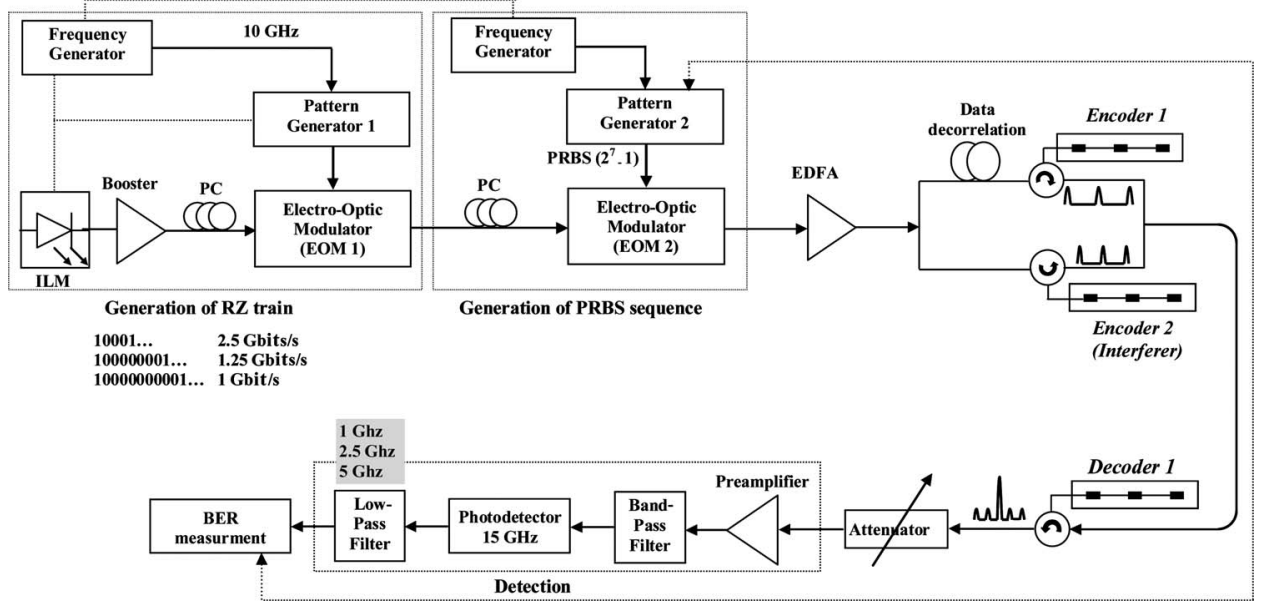

Fig. 4. Bit-error-rate experimental setup. 


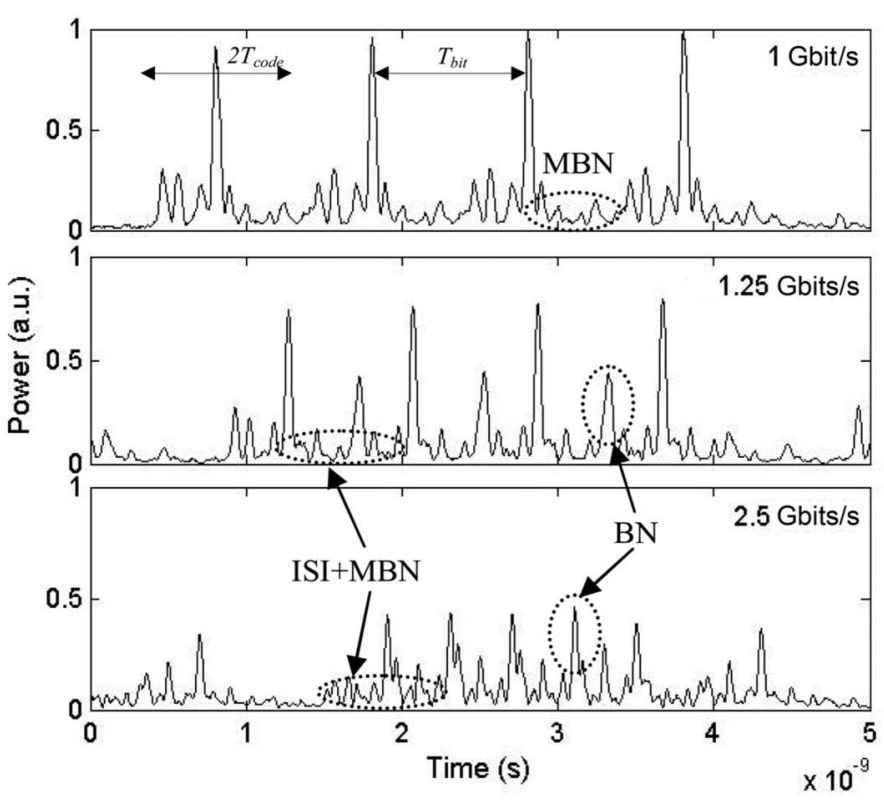

(a)

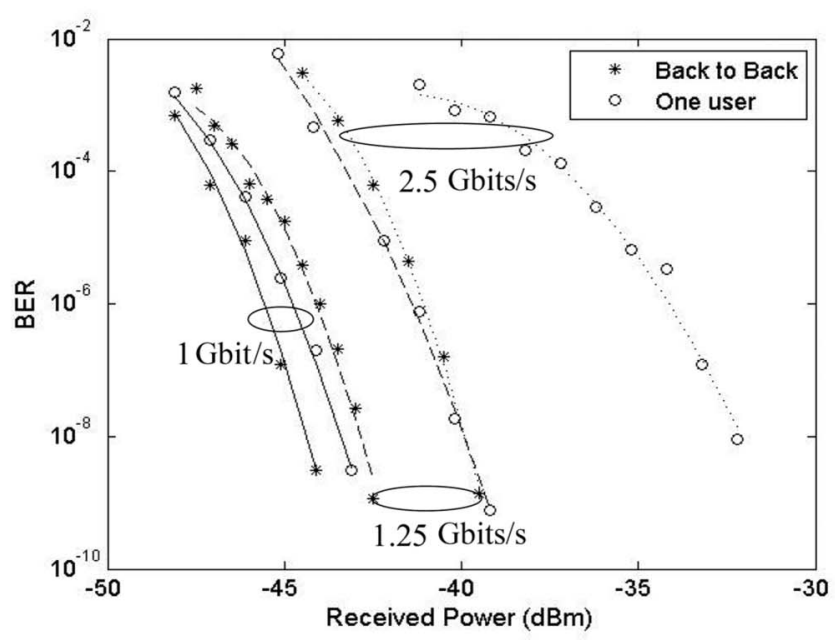

(b)

Fig. 5. (a) Decoded signals for $1 \mathrm{Gbit} / \mathrm{s}, 1.25 \mathrm{Gbits} / \mathrm{s}$, and $2.5 \mathrm{Gbits} / \mathrm{s}$ data bit rates using a nonperiodic code PS $(L=9)$, (b) experimental BER measurements.

[Fig. 6(a)]. A 10 $0^{-9}$ BER transmission is achieved when using EQC codes for $1 \mathrm{Gbit} / \mathrm{s}$ and $1.25 \mathrm{Gbits} / \mathrm{s}$. We note, however, an error floor in the two-user configuration, appearing at a $2.5 \mathrm{Gbits} / \mathrm{s}$ data bit rate due to the accumulation of MBN, ISI, and MAI [Fig. 6(b)].

Comparing Figs. 5 and 6, we note that the ratio between the amplitude of the main peak of the autocorrelation and the maximum level of the out-of-phase sidelobes depends on the code family. Because of their good correlation properties, out-of-phase autocorrelation sidelobes of EQC codes are lower than out-of-phase sidelobes of PS codes, i.e., sparser codes allow a higher optical signal-to-noise ratio at detection to be achieved. Under those last conditions, threshold detection will be easier and BER performance better.

\section{B. Receiver Bandwidth Constraint Analysis}

In a low-cost optical access network concept, using a low-speed photodetector is more suitable, and this means that the receiver integration time duration should be as close as possible to the bit time duration. However, in a DS-OCDMA system, this integra- 


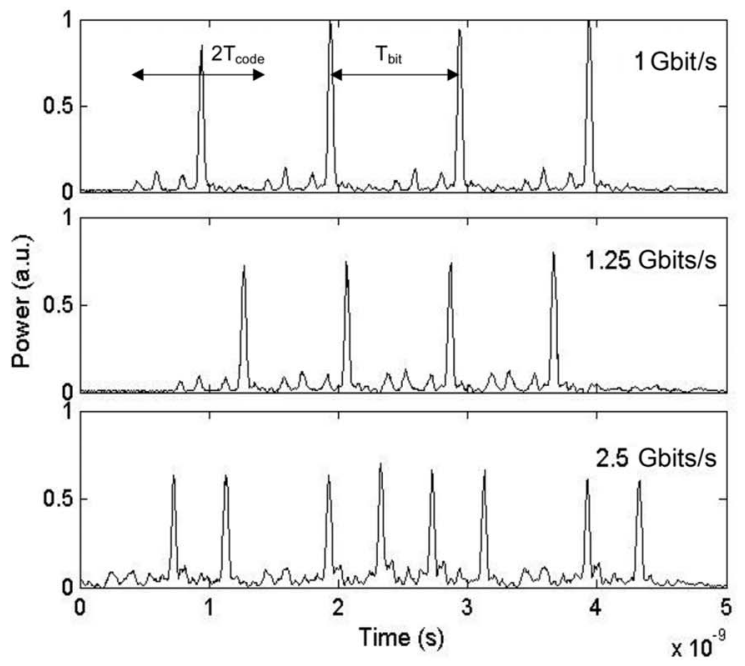

(a)
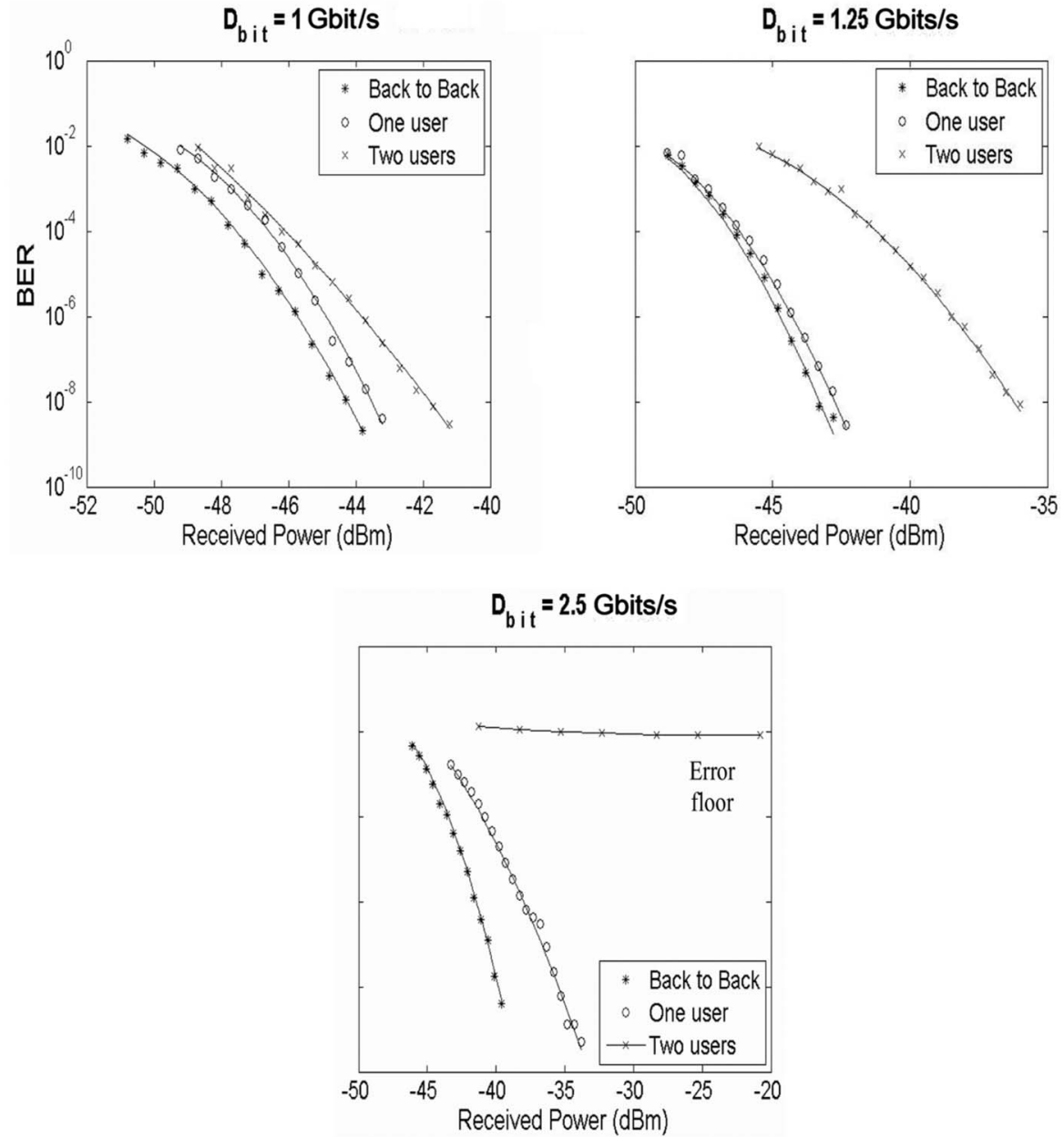

(b)

Fig. 6. (a) Decoded signals for $1 \mathrm{Gbit} / \mathrm{s}, 1.25 \mathrm{Gbits} / \mathrm{s}$, and $2.5 \mathrm{Gbits} / \mathrm{s}$ data bit rates using a nonperiodic code EQC $(L=15)$, (b) experimental BER measurements.

tion time duration has to be equal to the chip time duration in order to reconstruct perfectly the autocorrelation function. We have studied the effect on the performance of the DS-OCDMA system when relaxing this constraint. To evaluate the performance degradations related to the receiver bandwidths, we have used electrical low-pass fil- 

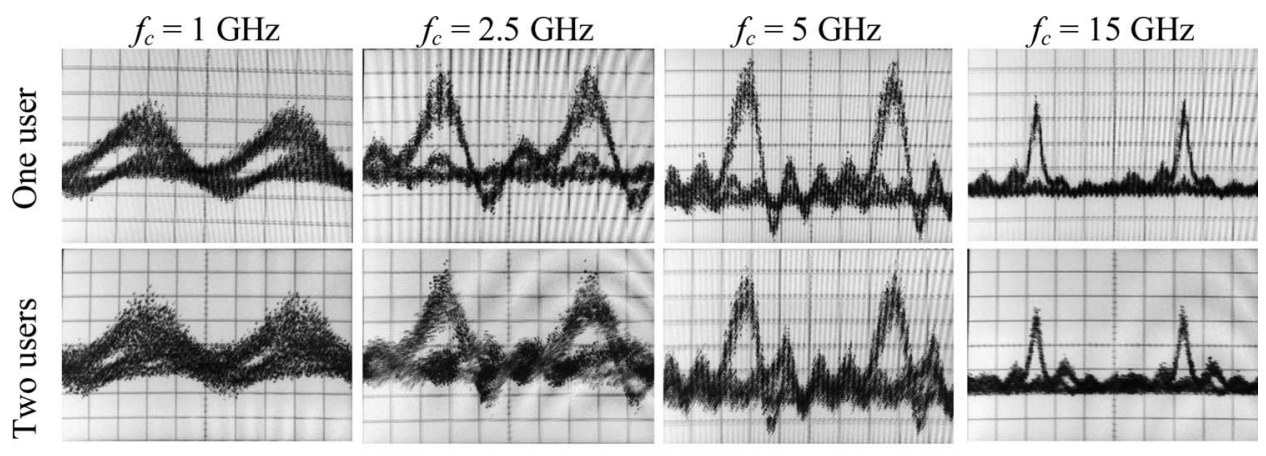

for $f_{c}=15 \mathrm{GHz}: 50 \mathrm{mV} / \mathrm{div}$, for other $f_{c}, 20 \mathrm{mV} / \mathrm{div}$.

Fig. 7. Experimental eye diagrams observed for $1,2.5,5$, and $15 \mathrm{GHz}$ values of the receiver bandwidths.

ters after the photodetector, which have permitted us to sweep the integration time duration of the receiver from the chip time duration up to the bit time duration.

Measured eye diagrams are analyzed for different cutoff frequencies (Fig. 7). Good performance is obtained in one- and two-user configurations for 15 and $5 \mathrm{GHz}$ receiver bandwidths. Using a $15 \mathrm{GHz}$ receiver bandwidth corresponds approximately to the inverse of the chip time duration, and in that configuration, no broadening of the decoded pulses occurs. As the receiver bandwidth is decreased to $5 \mathrm{GHz}$, pulses broaden, but detection remains possible under good conditions. With lower bandwidths (2.5 and $1 \mathrm{GHz}$ ), eye diagrams are degraded, and the analysis of BER performance reveals power penalties observed in the one-user configuration and two-user configuration (Fig. 8).

When the receiver bandwidth is approximately equal to the inverse of the bit time duration, i.e., $1 \mathrm{GHz}$, decoded pulses strongly broaden, leading to further BER error floor. In that case, to improve the signal-to-noise ratio and consequently the BER performance of the system, optical thresholding techniques have to be used to reduce MAI noise. Various techniques have been studied for this purpose, such as using highly nonlinear fiber (HNLF), supercontinuum generation in dispersion-flattened fiber, and a nonlinear optical-loop mirror (NOLM) [14-16].

\section{Conclusion}

In this paper, we have pointed out that an in-depth analysis of the code structure allows the optimization of the behavior of a coherent DS-OCDMA system. In the DS-OCDMA system studied here, encoders and decoders based on superstructured

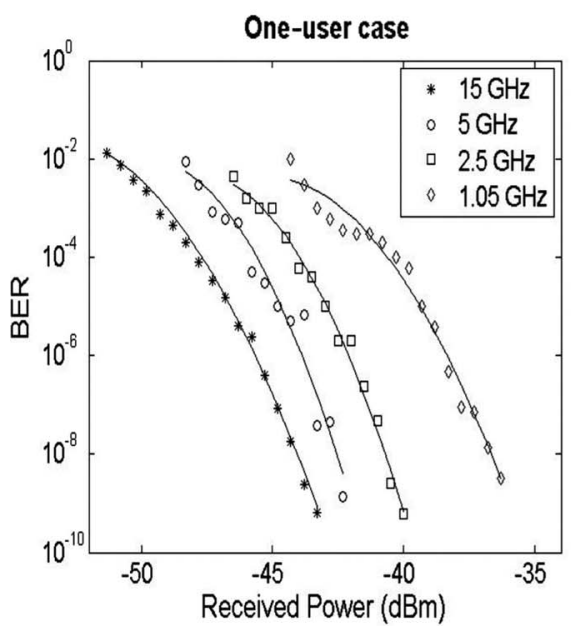

(a)

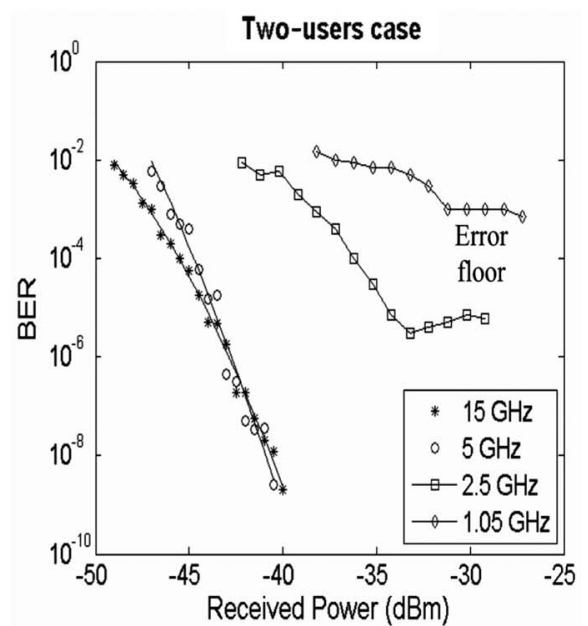

(b)

Fig. 8. Measured BER performance at $1 \mathrm{Gbit} / \mathrm{s}$ using $1,2.5,5$, and $15 \mathrm{GHz}$ receiver bandwidths: (a) one-user case, (b) two-user case. 
fiber Bragg gratings behave spuriously as multipath interferometers. This behavior together with the coherence time of the laser source leads to MBN as soon as primary and multiple reflected pulses superimpose within one chip time duration and interfere. These coherence time impairments have been demonstrated to be strongly related to the periodicity of the structure of the implemented codes.

To improve the robustness of this coherent DS-OCDMA system, we consider here nonperiodic $\mathrm{QC}$ and $\mathrm{EQC}$ codes. We have measured the experimental temporal responses of the encoder and decoder and have obtained a good agreement between simulations and experiments. We have demonstrated that encoder and decoder temporal responses are less sensitive to source coherence time impairments and consequently the measured autocorrelation functions are more robust.

Finally, we have demonstrated that sparse and nonperiodic EQC codes strongly improve the BER performance of the coherent DS-OCDMA system. The good correlation properties due to the sparse structure of the EQC codes have been shown to reduce ISI in comparison with PS codes. The nonperiodicity of the structure of the EQC codes have been demonstrated to diminish MBN. The relative low amplitude level of ISI and MBN with EQC codes let us also increase the signal-to-noise ratio of the autocorrelation function. With this feature, the threshold technique will operate more easily than with PS codes in order to eliminate MAI. At last, we have shown that the reduction of ISI and MBN observed with EQC codes allows the constraints on the receiver bandwidth to be relaxed from 15 to $5 \mathrm{GHz}$ without any BER penalty, making the detection of the DS-OCDMA system less expensive.

\section{References}

1. J. A. Salehi, "Emerging OCDMA communication systems and data networks," J. Opt. Netw. 6, 1138-1178 (2007).

2. S. J. B. Yoo, J. P. Heritage, V. Hernandez, R. Scott, W. Cong, N. Fontaine, R. Broeke, J. Cao, S.-W. Seo, J.-H. Baek, F. Soares, Y. Du, C. Yang, W. Jiang, K. Aihara, Z. Ding, B. Kolner, A.-V. Pham, S. Lin, F. Olsson, Y. Sun, S. Lourdudoss, K. Y. Liou, S. N. G. Chu, R. Hamm, B. Patel, W. S. Hobson, J. R. Lothian, S. Vatanapradit, L. Gruezke, W. T. Tsang, M. Shearn, and A. Scherer, "Spectral phase encoded time spread optical code division multiple access technology for next generation communication networks [Invited]," J. Opt. Netw. 6, 1210-1227 (2007).

3. J. Penon, Z. El Sahn, L. A. Rusch, and S. LaRochelle, "Spectral-amplitude-coded OCDMA optimized for a realistic FBG frequency response," J. Lightwave Technol. 25, 1256-1263 (2007).

4. C.-S. Brès, I. Glesk, and P. R. Prucnal, "Demonstration of an eight-user 115-Gchip/s incoherent OCDMA system using supercontinuum generation and optical time gating," IEEE Photon. Technol. Lett. 18, 889-891 (2006).

5. S. M. R. Motaghian Nezam, P. Saghari, A. E. Willner, R. Omrani, and P. Vijay Kumar, "Experimental demonstration of OCDMA transmission using a three-dimensional (timewavelength-polarization) codeset," J. Lightwave Technol. 23, 3282-3289 (2005).

6. L. Tancevski and L. A. Rush, "Impact of beat noise on the performance of 2-D optical CDMA systems," IEEE Commun. Lett. 4, 264-266 (2000).

7. S. Shen, A. M. Weiner, G. D. Sucha, and M. L. Stock, "Bit error rate performance of ultrashort-pulse optical OCDMA detection under multi-access interferences," Electron. Lett. 36, 1795-1796 (2002).

8. X. Wang and K. Kitayama, "Analysis of beat noise in coherent and incoherent timespreading OCDMA,” J. Lightwave Technol. 22, 2226-2235 (2004).

9. I. Fsaifes, C. Lepers, M. Lourdiane, P. Gallion, V. Beugin, and P. Guignard, "Source coherence impairments in a direct detection direct sequence optical code division multiple access system," Appl. Opt. 46, 456-462 (2007).

10. S. V. Maric, "New family of algebraically designed optical orthogonal codes for use in CDMA fiber optic networks," Electron. Lett. 29, 538-539 (1993).

11. C.-S. Brès, Y.-K. Huang, D. Rand, I. Glesk, P. R. Prucnal, T. Bazan, C. Michie, D. Harle, and I. Andonovic, "On the experimental characterization of beat noise in 2-D time-spreading wavelength-hopping OCDMA systems," IEEE Photon. Technol. Lett. 18, 2314-2316 (2006).

12. R. Adams and L. R. Chen, "Effect of encoder-decoder mismatch due to wavelength and time misalignments on the performance of two-dimensional wavelength-time optical code division multiple access systems," Appl. Opt. 44, 4368-4374 (2005).

13. I. Fsaifes, C. Lepers, A.-F. Obaton, and P. Gallion, "DS-OCDMA encoder/decoder performance analysis using optical low coherence reflectometry," J. Lightwave Technol. 24, 3121-3128 (2006).

14. R. P. Scott, K. Li, W. Cong, V. J. Hernandez, B. H. Kolner, J. P. Heritage, and S. J. B. Yoo, "Demonstration of an error-free 4/spl times/10 Gb/s multiuser SPECTS O-CDMA network testbed," IEEE Photon. Technol. Lett. 16, 2186-2188 (2004).

15. X. Wang, N. Wada, and K.-I. Kitayama, "Performance degradation in coherent OCDMA due to receivers bandwidth limit and improvement by using optical thresholding," in The 18th 
Annual Meeting of the IEEE Lasers and Electro-Optics Society (IEEE, 2005), pp. 638-639.

16. J. H. Lee, P. C. Teh, P. Petropoulos, M. Ibsen, and D. J. Richardson, "A grating-based OCDMA coding-decoding system incorporating a nonlinear optical loop mirror for improved code recognition and noise reduction," J. Lightwave Technol. 20, 36-46 (2002). 